Portland State University

PDXScholar

\title{
A Better Nondimensionalization Scheme for Slender Laminar Flows: The Laplacian Operator Scaling Method
}

\author{
Mark M. Weislogel \\ Portland State University, weisloge@pdx.edu \\ Yongkang Chen \\ Portland State University \\ D. Bolleddula \\ Portland State University
}

Follow this and additional works at: https://pdxscholar.library.pdx.edu/mengin_fac

Part of the Fluid Dynamics Commons, and the Materials Science and Engineering Commons Let us know how access to this document benefits you.

\section{Citation Details}

Weislogel, M. M., Chen, Y. Y., \& Bolleddula, D. D. (2008). A better nondimensionalization scheme for slender laminar flows: The Laplacian operator scaling method. Physics Of Fluids, 20(9), 093602.

This Article is brought to you for free and open access. It has been accepted for inclusion in Mechanical and Materials Engineering Faculty Publications and Presentations by an authorized administrator of PDXScholar. Please contact us if we can make this document more accessible: pdxscholar@pdx.edu. 


\title{
A better nondimensionalization scheme for slender laminar flows: The Laplacian operator scaling method
}

\author{
M. M. Weislogel, ${ }^{\text {a) }}$ Y. Chen, ${ }^{\text {b) }}$ and D. Bolleddula ${ }^{\text {c) }}$ \\ Engineering Building, Room 401, Portland State University, 1930 SW 4th Avenue, Portland, \\ Oregon 97201, USA
}

(Received 30 October 2007; accepted 23 July 2008; published online 2 September 2008)

\begin{abstract}
A scaling of the two-dimensional Laplacian operator is demonstrated for certain solutions (at least) to Poisson's equation. It succeeds by treating the operator as a single geometric scale entity. The belated and rather subtle method provides an efficient assessment of the geometrical dependence of the problem and is preferred when practicable to the hydraulic diameter or term-by-term scaling for slender fully developed laminar flows. The improved accuracy further reduces the reliance of problems on widely varying numerical data or cumbersome theoretical forms and improves the prospects of exact or approximate theoretical analysis. Simple example problems are briefly described that demonstrate the application and potential of the method. (C) 2008 American Institute of Physics. [DOI: 10.1063/1.2973900]
\end{abstract}

\section{THE LAPLACIAN OPERATOR SCALING METHOD}

Considering the Cartesian velocity field $\boldsymbol{u}=(u, v, w)$, the equation governing low-inertia, weakly time-dependent, and predominantly $z$-directional flows is a Poisson equation derived from the $z$-component momentum equation:

$$
k=\Delta w=\left(\frac{\partial^{2}}{\partial x^{2}}+\frac{\partial^{2}}{\partial y^{2}}+\frac{\partial^{2}}{\partial z^{2}}\right) w,
$$

subject to boundary conditions. As is often helpful, assuming acceptable scales for $x, y, z$ and $k$ can be identified for the problem, scale analysis may be performed on Eq. (1) to determine the velocity scale for $w$ with which Eq. (1) can in turn be nondimensionalized. In situations where the "crossflow coordinates" $x$ and $y$ can be parametrized and scaled in terms of $z$ and $t$, it is proposed that such spatially and temporally dependent length scales $x \sim \bar{x}_{s}$ and $y \sim \bar{y}_{s}$ with $z \sim z_{s}$ $=L$ may be employed to compute a spatially and temporally dependent velocity scale $w \sim \bar{w}_{s}$. Provided the flow is slender, $\left(\bar{x}_{s} / L\right)^{2} \ll 1$, treating $\Delta$ as a single scalable quantity such that $\Delta \sim \bar{\Delta}_{s}$, "scale analysis" on Eq. (1) is performed to determine

$$
w \sim \bar{w}_{s}=\frac{k}{\bar{\Delta}_{s}} \equiv \frac{k}{\frac{1}{\bar{x}_{s}^{2}}+\frac{1}{\bar{y}_{s}^{2}}} \equiv \frac{k \bar{x}_{s}^{2}}{1+\bar{T}_{s x y}^{2}},
$$

where $\bar{T}_{s x y}=\bar{x}_{s} / \bar{y}_{s}$. The overbar for $\bar{x}_{s}$ and $\bar{y}_{s}$ denotes local, potentially $z$ - and/or $t$-dependent $x$ - and $y$-coordinate length scales such that, in general $\bar{x}_{s}=\bar{x}_{s}(z, t), \bar{y}_{s}=\bar{y}_{s}(z, t), L=L(t)$, $k=k(z, t)$, and thus $\bar{w}_{s}=\bar{w}_{s}(z, t)$. The method introduces a notation $\bar{\Delta}_{s}$ for the local $z$ - and $t$-dependent Laplacian operator scale which is treated as its own term-a minor twist on the

\footnotetext{
${ }^{a)}$ Electronic mail: mmw@cecs.pdx.edu.

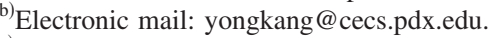

${ }^{c}$ Electronic mail: dabolla@cecs.pdx.edu. Telephone: 503-725-4290. Fax: 503-725-8255.
}

more common and intuitive term-by-term scaling method. ${ }^{1}$ The "Laplacian scale" $\bar{\Delta}_{s}$ obeys commutative laws (e.g., $\bar{\Delta}_{s} \bar{w}_{s}=\bar{w}_{s} \bar{\Delta}_{s}$ ) - it is not an operator but the scale of an operator. Using velocity scale (2) and spatially dependent length scales, Eq. (1) when nondimensionalized becomes

$$
1=\frac{1}{\left(1+\bar{T}_{s x y}^{2}\right)} \frac{\partial^{2} \bar{w}^{*}}{\partial \bar{x}^{* 2}}+\frac{\bar{T}_{s x y}^{2}}{\left(1+\bar{T}_{s x y}^{2}\right)} \frac{\partial^{2} \bar{w}^{*}}{\partial \bar{y}^{* 2}},
$$

where $\bar{x}^{*}=x / \bar{x}_{s}, \bar{y}^{*}=y / \bar{y}_{s}$, and $\bar{w}^{*}=w \bar{\Delta}_{s} / k=w\left(1+\bar{T}_{s x y}^{2}\right) / k \bar{x}_{s}^{2}$. The result of Eq. (3) is forwarded as a modified twodimensional (2D) Poisson equation that, despite having $z$ and $t$-dependent variables and coefficients, can lead to narrower bounds for numerical coefficients for the areaaveraged velocity,

$$
\left\langle\bar{w}^{*}\right\rangle=\frac{\bar{x}_{s} \bar{y}_{s}}{A} \iint \bar{w}^{*} d \bar{x}^{*} d \bar{y}^{*} \equiv F_{i},
$$

where $A$ is the dimensional section area. We do not make any significant effort to examine (or prove) the generality of this claim. Instead we demonstrate the usefulness of the approach with several basic example problems where $\bar{\Delta}_{s}=$ const, $\bar{\Delta}_{s}$ $=\bar{\Delta}_{s}(z)$, and $\bar{\Delta}_{s}=\bar{\Delta}_{s}(z, t)$.

\section{A. Steady fully developed laminar flow in a rectangular duct: $\bar{\Delta}_{s}=$ const $=\Delta_{s}$}

A schematic of this historic flow is provided in Fig. 1 with coordinates and characteristic lengths identified. It is frequently desirable to quickly determine the average velocity of the flow through the duct. Applying the present scaling approach, noting that the length scales for the duct are simply constants, $\bar{x}_{s}=a, \bar{y}_{s}=b$, and $\bar{z}_{s}=L$, where $(a / L)^{2} \ll 1$, the local dimensional velocity scale from Eq. (2) may be written directly, 


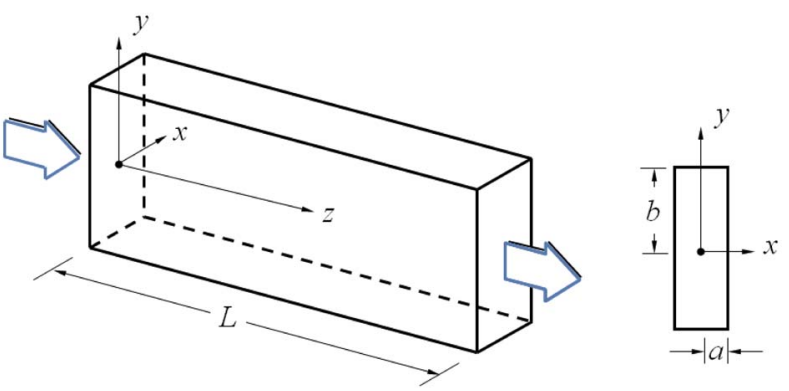

FIG. 1. (Color online) Schematic of rectangular duct geometry; section at right.

$$
\begin{aligned}
\bar{w}_{s}=\frac{k}{\bar{\Delta}_{s}} & =-\frac{P_{z}}{\mu} \frac{1}{\left(\frac{1}{a^{2}}+\frac{1}{b^{2}}\right)} \\
& =-\frac{P_{z}}{\mu} \frac{\bar{x}_{s}^{2}}{\left(1+\bar{T}_{s x y}^{2}\right)} \\
& =-\frac{P_{z}}{\mu} \frac{a^{2}}{\left(1+a^{2} / b^{2}\right)},
\end{aligned}
$$

where $k=P_{z} / \mu$, with $P_{z}$ the pressure gradient along $z$ and $\mu$ the dynamic viscosity of the fluid. (Subscript notation for partial differentiation is only implied herein for $z$ and $t$.) The negative sign in Eq. (5) assures $\bar{w}_{s}>0$ when $P_{z}<0$. As reviewed by Shah and London (Ref. 2, Chap. 7A), when the hydraulic diameter scaling is applied to this problem, $x_{s}=y_{s}$ $\sim a b /(a+b)$, and term-by-term similar scaling of Eq. (1) produces

$$
\bar{w}_{\text {hyd }}=-\frac{P_{z}}{\mu} \frac{a^{2}}{(1+a / b)^{2}} .
$$

The exact analytic solution for the average velocity, ${ }^{2}$

$$
\begin{aligned}
\langle w\rangle & =-\frac{P_{z}}{\mu} \frac{\bar{x}_{s}^{2}}{3}\left[1-\frac{192}{\pi^{5}} \bar{T}_{s x y} \sum_{i=1,3,5, \ldots}^{\infty} \frac{\tanh \left(i \pi / 2 \bar{T}_{s x y}\right)}{i^{5}}\right] \\
& \equiv F_{i} \bar{w}_{s} \\
& \equiv F_{\text {ihyd }} \bar{w}_{\text {hyd }},
\end{aligned}
$$

is normalized by both the Laplacian [Eq. (5)] and hydraulic diameter [Eq. (6)] velocity scales and plotted against duct aspect ratio $a / b$ in Fig. 2. Both approaches capture the order of magnitude of the exact average velocity for all $a$ and $b$. However, the Laplacian scaling delivers at most a significant fourfold reduction in the variance of $F_{i}$ versus $F_{i \text { hyd }}$.

The result of Eq. (5) is similar in form to that presented by Bejan [Ref. 3, Eq. (3.33)] following integration of assumed parabolic velocity fields expressed in terms of the cross-flow coordinates. However, Eq. (5) is here determined directly and simply by $\bar{\Delta}_{s}$ and provides a quick, concise, and more geometrically representative approximation of $\langle w\rangle$ for use in subsequent analytical solutions that are consequently only weakly dependent on further cumbersome solution forms or numerical data. The difference between Eqs. (5) and (6) is subtle for this problem, but disparities can increase

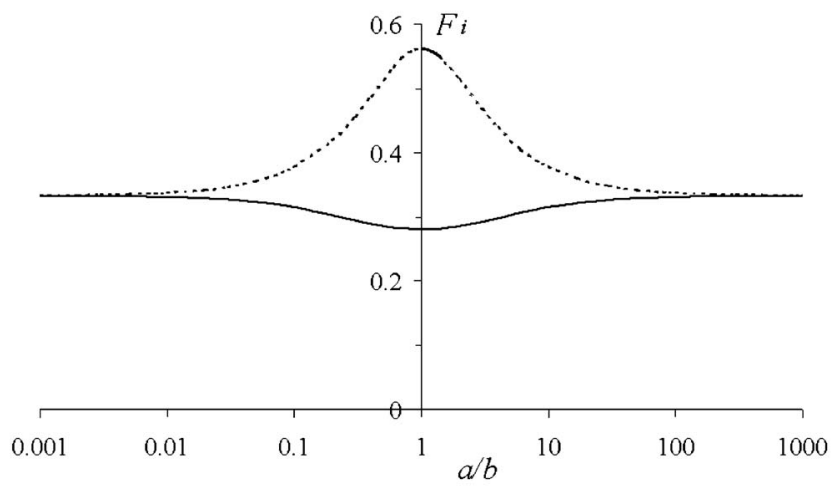

FIG. 2. Comparison of exact solution for $\langle w\rangle$ [Eq. (7)] normalized by the Laplacian scale velocity [solid line, Eq. (5), $F_{i}$ ] and hydraulic diameter [dashed line, Eq. (6), $F_{\text {ihyd }}$.

dramatically in favor of the Laplacian scaling method for problems of increased geometric complexity. ${ }^{4}$ Equality between $\langle w\rangle$ and $\bar{w}_{s}$ is established in Eq. (7) by introducing the narrowly varying function $F_{i}$, also defined in Eq. (4); for the rectangular section, $0.281 \ldots \leq F_{i}(a / b) \leq 1 / 3$. The fact that $F_{i} \approx 1 / 3$ with at worst a $15.6 \%$ shortfall opens up further possibilities for approximate analytical solutions.

Defined in this manner, the function $F_{i}$ is inversely proportional to previously determined friction factors. ${ }^{2,5,6}$ For the rectangular section ${ }^{2}$ also reviewed by White, ${ }^{7}$ it can be shown that the Poiseuille number is confined such that 14.2... $\leq \mathrm{Po} \equiv f \operatorname{Re}=8\left(1+a^{2} / b^{2}\right) / F_{i}(1+a / b)^{2} \leq 24$, where $f$ is the Fanning friction factor with hydraulic diameter Reynolds number $\operatorname{Re}=\rho U D_{\text {hyd }} / \mu$, where $D_{\text {hyd }}=4 A_{s} / P$, with $A_{s}$ and $P$ as the total section area and wetted perimeter, respectively. Not only is $F_{i}$ or its inverse four times more confined than Po but it is $O(1)$. Last, using the $\bar{\Delta}_{s}$ scaling it can be shown that if a single viscous length $r_{\mu}$ was computed, characterizing the average velocity for this flow, it would be $r_{\mu}$ $=\left(2 F_{i} / \bar{\Delta}_{s}\right)^{1 / 2} \approx(2 / 3)^{1 / 2} a b /\left(a^{2}+b^{2}\right)^{1 / 2}$. For such laminar flows this "viscous length" is accurate, satisfying $\langle w\rangle$ $\approx P_{z} r_{\mu}^{2} / 2 \mu$, and is more akin to a section area divided by a section diagonal as opposed to a section area divided by a section perimeter (i.e., the hydraulic diameter approach). Note that Po may be converted to $F_{i}$ using $F_{i}=D_{\text {hyd }}^{2} \bar{\Delta}_{s} / 2$ Po .

\section{B. Constant rate laminar flow in a slender constant area rectangular duct of varying aspect ratio: $\bar{\Delta}_{s}=\bar{\Delta}_{s}(z)$}

The accommodation of spatially varying scales can be demonstrated with the pedagogical problem sketched in Fig. 3. For this duct, $\langle w\rangle=\mathrm{const}=\left\langle w_{o}\right\rangle$ and $A=\mathrm{const}=4 a b$. As sketched, for a linearly tapering duct in the $y-z$ plane, the duct boundaries are defined by $x_{w}= \pm a b /(b-m z)$ and $y_{w}$ $= \pm(b-m z)$, where $m=(b-a) / L$.

One may want to quickly compute $P_{z}$ or $P(z)$ for this flow in instances where $\left\langle w_{o}\right\rangle$ is known. Using the Eq. (7) definition $\langle w\rangle \equiv F_{i} \bar{w}_{s}$, Eq. (2) is employed again but now with $z$-dependent length scales. For example, when $(a / L)^{2}$ 

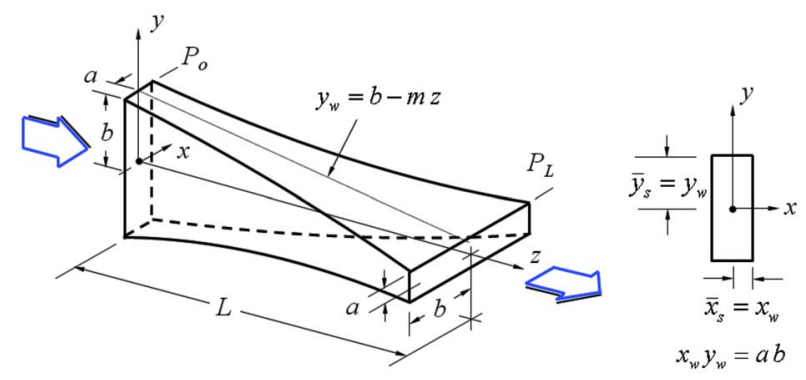

FIG. 3. (Color online) Schematic of varying aspect ratio constant area rectangular duct (not to scale); section at right.

$\ll 1$, substituting the $z$-dependent length scales $\bar{x}_{s}=x_{w}$ and $\bar{y}_{s}=y_{w}$ into Eq. (2) and noting $\bar{T}_{s x y}=a b /(b-m z)^{2}$, solving for $P_{z}$ yields

$$
P_{z}=-\frac{\mu\left\langle w_{o}\right\rangle}{F_{i}}\left(\frac{b-m z}{a b}\right)^{2}\left(\frac{a^{2} b^{2}}{(b-m z)^{4}}+1\right) .
$$

However, $F_{i} \approx 1 / 3$ and is a weak function of $z$. Treating $F_{i}$ $=1 / 3$ and applying the boundary condition $P(L)=P_{L}$, Eq. (8) may be integrated to find

$$
\begin{aligned}
P_{s} \approx & P_{L}-\mu\left\langle w_{o}\right\rangle \\
& \times\left[\frac{3 a^{2} b^{2}+\left(a^{3}-3 a b^{2}\right)(b-m z)-(b-m z)^{4}}{a^{2} b^{2} m(b-m z)}\right],
\end{aligned}
$$

which is more accessible than the exact solution

$$
\begin{aligned}
P_{e}= & -3 \mu\left\langle w_{o}\right\rangle \int\left(\frac{b-m z}{a^{2} b^{2}}\right)\left\{1-\frac{192}{\pi^{5}} \frac{a b}{(b-m z)^{2}}\right. \\
& \left.\times \sum_{i=1,3,5, \ldots}^{\infty} \frac{\tanh [i \pi(b-m z) / 2 a b]}{i^{5}}\right\}^{-1} d z,
\end{aligned}
$$

subject also to $P(L)=P_{L}$, which must be integrated numerically. [Subscripts $s$ and $e$ denote "scale" and "exact" forms for pressure $P(z)$.] Choosing $P_{L}=0$, from Eq. (9) the upstream pressure is

$$
P_{s}(0)=\mu\left\langle w_{o}\right\rangle L\left(\frac{a^{2}+4 a b+b^{2}}{a^{2} b^{2}}\right) .
$$

Using this pressure to nondimensionalize Eqs. (9) and (10) yields the respective dimensionless pressure distributions

$$
P_{s}^{*}\left(z^{*}\right)=\frac{\left[b-(b-a) z^{*}\right]^{4}-\left(a^{3}-3 a b^{2}\right)\left[b-(b-a) z^{*}\right]-3 a^{2} b^{2}}{(b-a)\left(a^{2}+4 a b+b^{2}\right)\left[b-(b-a) z^{*}\right]}
$$

and

$$
\begin{aligned}
P_{e}^{*}\left(z^{*}\right)= & -3 \int \frac{\left[b-(b-a) z^{*}\right]^{2}}{\left(a^{2}+4 a b+b^{2}\right)}\left\{1-\frac{192}{\pi^{5}} \frac{a b}{\left[b-(b-a) z^{*}\right]^{2}}\right. \\
& \left.\times \sum_{i=1,3,5, \ldots}^{\infty} \frac{\tanh \left\{i \pi\left[b-(b-a) z^{*}\right]^{2} / 2 a b\right\}}{i^{5}}\right\} d z^{*},
\end{aligned}
$$

where $z^{*}=z / L$. Lengths $a$ and $b$ are nondimensionalized by $A^{1 / 2} / 2$, such that in dimensionless form $b^{*}=a^{*-1}$. The functions of Eqs. (12) and (13) are compared in Fig. 4(a) for a selection of values for $a^{*}$. The agreement is complete for $a^{*} \ll 1$ and $a^{*} \gg 1$ and worsens to at most $15 \%$ as $a^{*}$ approaches 1 . Any discrepancy at all is due to $F_{i}$ being treated as a constant in Eq. (9). As will be presented in connection with Figs. 7 and 9, it can be shown that a simple correlation for $F_{i}\left(a^{*}\right)$ is $F_{i} \approx\left(1-\frac{1}{6} \sin 2 \alpha\right) / 3$, where $\alpha$ for the rectangular section is defined as $\alpha=\tan ^{-1}(a / b)=\tan ^{-1} a^{* 2}$. Exploiting this relationship in Eq. (8) leads to

$$
P_{c}^{*}\left(z^{*}\right)=\frac{P_{s}^{*}\left(z^{*}\right)}{\left(1-\frac{1}{6} \sin 2 \alpha\right)},
$$

where subscript $c$ denotes a "correlated" scale value. $P_{c}^{*}\left(z^{*}\right)$ is also compared to $P_{e}^{*}\left(z^{*}\right)$ in Fig. 4(b), where conditions $a^{*} \ll 1, a^{*}=1$, and $a^{*} \gg 1$ agree completely with intermediate discrepancies $<5 \%$.

The simple $\bar{\Delta}_{s}$ scale solutions (12) and (14) do well in capturing the $z$ dependence of the flow with errors typically less than $15 \%$ and $5 \%$, respectively. Exact theoretical or numerical solutions are preferable, but in many cases such solutions mask the largely simple geometric dependence of the flow typified by such examples, i.e., Eq. (5) versus Eq. (7) and Eq. (9) versus Eq. (10). The compact forms resulting from the Laplacian scale approach further encourages the notion that the method might be exploited to greater effect in theoretical analyses where, say, $\langle w\rangle$ or $P$ become variables in subsequent derivations or models. A sample problem of this kind is highlighted below in three steps.

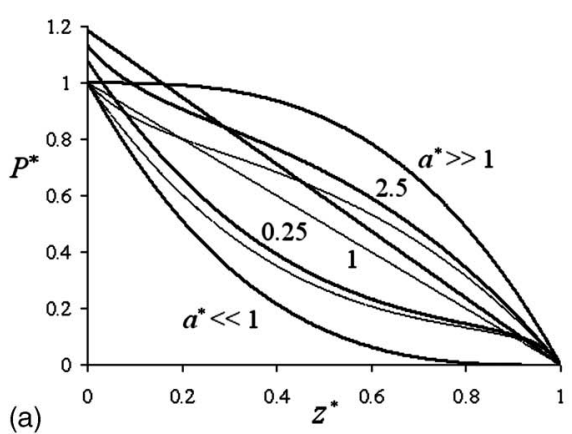

(a)

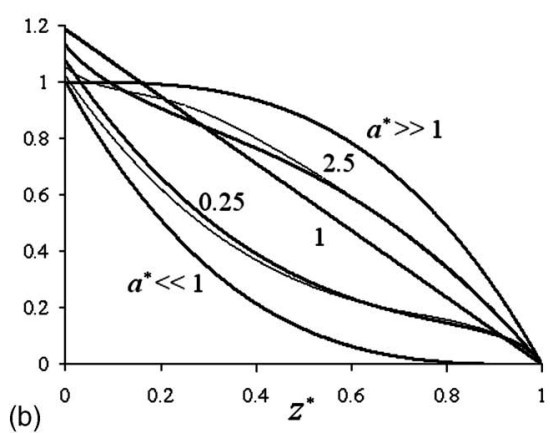

FIG. 4. (a) $P_{s}^{*}\left(z^{*}\right)$ (light) and $P_{e}^{*}\left(z^{*}\right)$ (heavy) for select values of $a^{*}$. Values for $a^{*} \ll 1$ and $a^{*} \ll 1$ coincide. (b) $P_{c}^{*}\left(z^{*}\right)$ (light) and $P_{e}^{*}\left(z^{*}\right)$ (heavy). Values for $a^{*} \ll 1, \quad a^{*} \gg 1$, and $a^{*}=1$ coincide. 

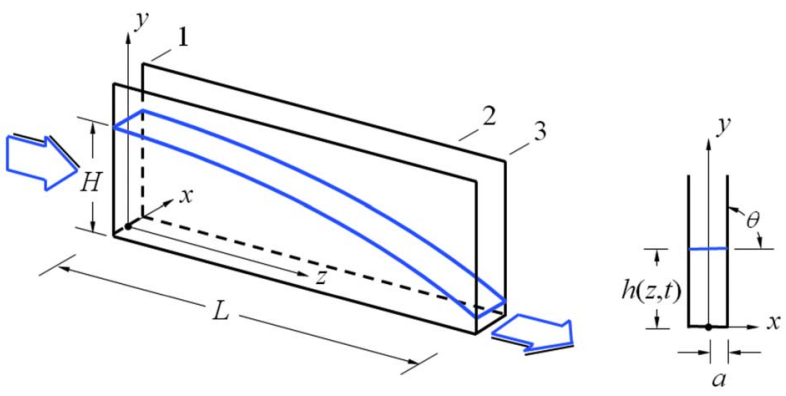

FIG. 5. (Color online) Schematic of gravity-driven flow along a rectangular channel; section at right (contact angle $\theta=\pi / 2$ ).

\section{Laminar gravity-driven flow along a slender channel: $\bar{\Delta}_{s}=\bar{\Delta}_{s}(z, t)$}

This contrived example flow illustrates how $z$ - and $t$-dependent scales within the Laplacian scale $\bar{\Delta}_{s}$ assist in the formulation of governing evolution equations.

\section{Rectangular channel section}

A schematic with critical dimensions for this free surface flow with negligible surface tension and $\theta=\pi / 2$ contact angle is provided in Fig. 5. The driving hydrostatic pressure gradient is $P_{z}=\rho g h_{z}$ with $\rho$ the density difference across the free surface and $g$ the gravitation in the negative $y$ direction, i.e., $\mathbf{g}=(0,-g, 0)$. It is desirable for this flow to determine the governing evolution equation using the Laplacian scaling method. From Fig. $5 \bar{x}_{s}=$ const $=a, \bar{y}_{s}=h \equiv h(z, t)$, and $z_{s}$ $=$ const $=L \neq f c n(t)$. For a slender column $(a / L)^{2} \ll 1$. Employing Eq. (5) and the definition of $F_{i}$, Eq. (7) becomes

$$
\langle w\rangle=F_{i} \bar{w}_{s}=-F_{i} \frac{P_{z}}{\mu} \frac{\bar{x}_{s}^{2}}{\left(1+\bar{T}_{s x y}^{2}\right)}=-F_{i} \frac{P_{z}}{\mu} \frac{a^{2}}{\left[1+(a / h)^{2}\right]} .
$$

Conservation of volume along the $z$ axis for this flow requires

$$
A_{t}=-(A\langle w\rangle)_{z}=-\left(A F_{i} \bar{w}_{s}\right)_{z} .
$$

In this case, $A=2 a h$ and substitution of Eq. (15) into Eq. (16) yields the dimensional nonlinear governing equation,

$$
h_{t}=\frac{\rho g a^{2}}{\mu}\left(\frac{F_{i} h h_{z}}{1+(a / h)^{2}}\right) \text {. }
$$

This form is exact provided $F_{i}$ from Eq. (7) is employed. However, as demonstrated in Fig. 2, since $F_{i} \approx 1 / 3$, a wieldy approximate solution form is obtained by absorbing $F_{i}$ and $\rho g a^{2} / \mu$ into the time scale should further theoretical analysis be pursued as will be momentarily.

It is noted that Eq. (17) is a dimensional evolution equation derived using local, weakly $z$ - and $t$-dependent scales via $\bar{\Delta}_{s}$ through $h=h(z, t)$. Because the local scales are unknown and variable, Eq. (17) must be normalized by global scales that are known and constant. For this simple flow the global scales are $x \sim a, h \sim H, z \sim L$, and $t \sim L / w_{s}$, where $w_{s}$ follows from $\bar{w}_{s}$ but incorporates the global scales in a global (note

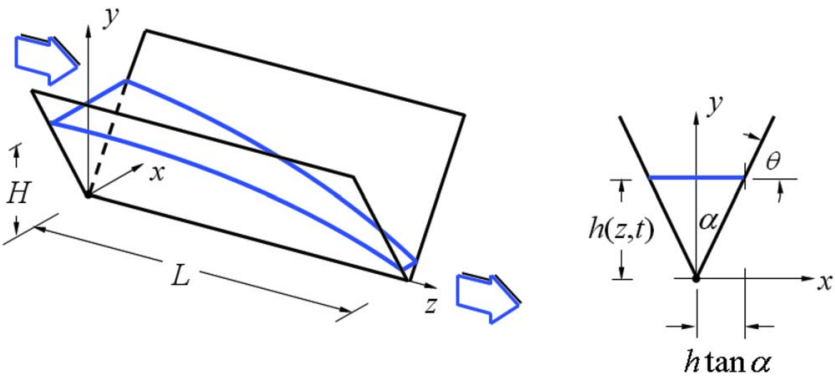

FIG. 6. (Color online) Schematic of gravity-driven flow in a triangular "groove" channel; section at right $(\theta=\pi / 2-\alpha)$.

the absence of overbar) Laplacian scale $\Delta_{s}=1 / a^{2}+1 / H^{2}$ such that

$$
w_{s} \sim \frac{P_{z}}{\mu \Delta_{s}} \sim \frac{P_{z s}}{\mu} \frac{1}{\left(\frac{1}{a^{2}}+\frac{1}{H^{2}}\right)} \sim \frac{\rho g H a^{2}}{\mu L\left(1+a^{2} / H^{2}\right)},
$$

where $P_{z s} \sim \rho g H / L$. Nondimensionalizing Eq. (17) now by the global scales yields the dimensionless nonlinear evolution equation

$$
h_{t^{*}}^{*}=\left(F_{i} \frac{\left(1+\lambda^{2}\right)}{1+\lambda^{2} / h^{* 2}} h^{*} h_{z^{*}}^{*}\right),
$$

where $\lambda \equiv a / H$. This form of the equation is $O(1)$ in all limits of the single parameter $\lambda^{2}$ since $F_{i}$ is approximately constant and $O(1)$. Equation (19) reveals different evolutionary behavior in the primary limits. For narrow channel flow, $\lambda^{2} / h^{* 2} \ll 1\left(F_{i} \approx 1 / 3\right)$,

$$
h_{t^{*}}^{*}=\left(F_{i} h^{*} h_{z^{*}}^{*}\right)_{z^{*}}
$$

For thin film flow, $\lambda^{2} / h^{* 2} \gg 1\left(F_{i} \approx 1 / 3\right)$,

$$
\lambda^{2} h_{t^{*}}^{*}=\left[F_{i}\left(1+\lambda^{2}\right) h^{* 3} h_{z^{*}}^{*}\right]_{z^{*}} .
$$

In essence, when $\lambda^{2} / h^{* 2} \ll 1$ the flow geometry transitions from a narrow channel flow [Eq. (20)], to the full flow [Eq. (19)], and then to a thin film flow [Eq. (21)] as $h^{*} \rightarrow 0$. This progression is qualitatively depicted by locations $1-3$ in Fig. 5 , respectively. The limiting equations (20) and (21) offer significantly improved opportunities to develop analytical solutions (exact, steady, similarity, etc.) to benchmark the governing equation (19), with the latter to be solved numerically in the majority. This result may be more appreciated for more geometrically complex flows as will be demonstrated.

\section{Triangular channel section}

This flow geometry is sketched in Fig. 6 for $\theta=\pi / 2-\alpha$ and is addressed identically as the rectangular channel flow only, $\bar{x}_{s}=h \tan \alpha, \bar{y}_{s}=h, z_{s}=L$, and $A=h^{2} \tan \alpha$. Again, taking $(h \tan \alpha / L)^{2} \ll 1$ and substitution of the $z$-dependent scales into Eq. (15) for $\langle w\rangle$ and then into Eq. (16), the governing equation for $0 \leq \alpha \leq \pi / 2$ becomes 


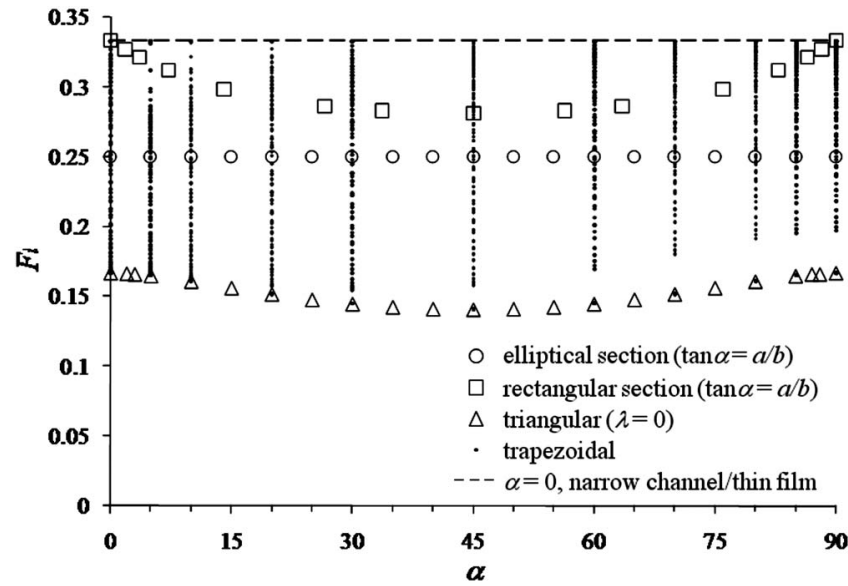

FIG. 7. Exact numerical values (dots) for $F_{i}$ in terms of $\alpha$ for trapezoidal section channel flow; limiting values rectangular and triangular sections are noted by symbols and dashed line (ellipse values provided for reference, $\left.F_{i}=1 / 4\right)$.

$$
\left(h^{2}\right)_{t}=\frac{\rho g \sin ^{2} \alpha}{\mu}\left(F_{i} h^{4} h_{z}\right)_{z} .
$$

An exact solution is not available for $F_{i}$ for all $\alpha$ for this section. However, numerical results in various forms ${ }^{2,5-8}$ are (in Ref. 2 see solutions for rhombi), the most recent of which are plotted in Fig. 7. It is found that for the triangular channel $\quad 0.1405 \ldots \leq F_{i}(\alpha) \leq 1 / 6, \quad$ or $\quad F_{i} \approx$ const $\approx 1 / 6-$ approximately $50 \%$ that of the rectangular channel, which is also plotted in Fig. 7 in terms of $\alpha$, where $\tan \alpha=a / b$. Nondimensionalizing Eq. (22) using the relevant global scales for this problem $x \sim H \tan \alpha, h \sim H, z \sim L$, and $t \sim L / w_{s}$, where $\Delta_{s}=1 / H^{2} \tan ^{2} \alpha+1 / 1 H^{2}=1 / H^{2} \sin ^{2} \alpha$, produces the governing evolution equation

$$
\left(h^{* 2}\right)_{t^{*}}=\left(F_{i} h^{* 4} h_{z^{*}}^{*}\right)_{z^{*}}
$$

\section{Trapezoidal channel}

In keeping with the schematic of Fig. 8 for this flow, $\bar{x}_{s}=(a+h \tan \alpha), \bar{y}_{s}=h, \bar{z}_{s}=L$, and $A=(2 a+h \tan \alpha) h$. Using global scales $x \sim a+H \tan \alpha, h \sim H, z \sim L$, and $t \sim L / w_{s}$ and taking $[(a+h \tan \alpha) / L]^{2} \ll 1$ produces the governing dimensionless equation
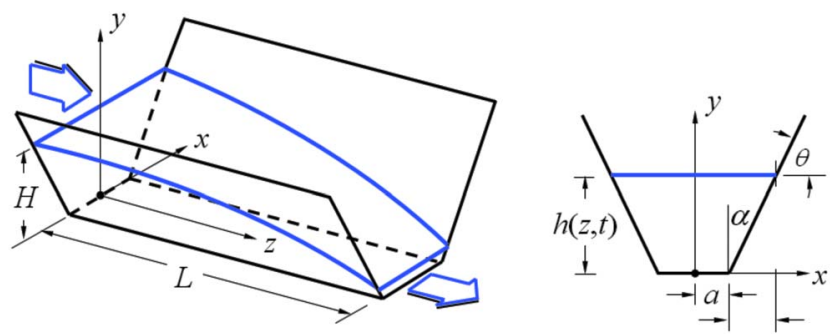

$h \tan \alpha$

FIG. 8. (Color online) Schematic of gravity-driven flow in a flattened triangular groove or "trapezoidal" section; section at right $(\theta=\pi / 2-\alpha)$.

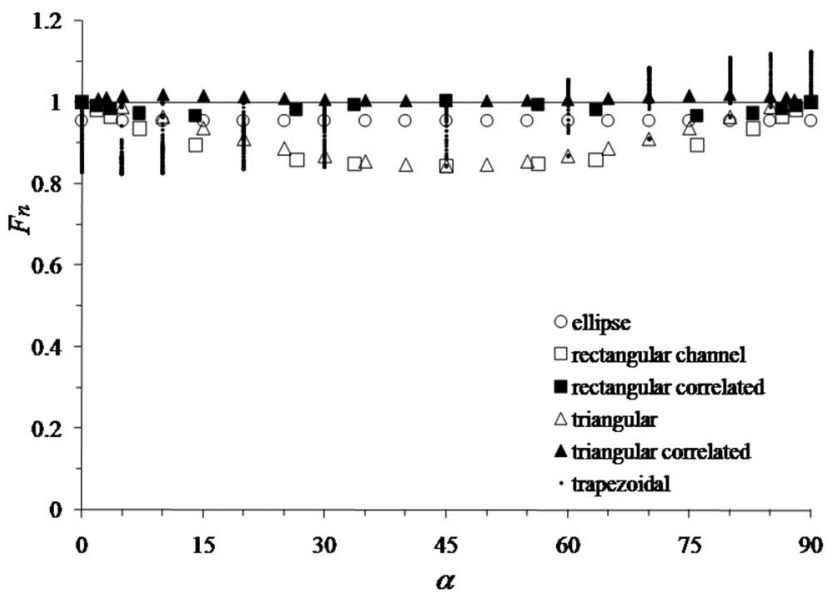

FIG. 9. $F_{n}(\alpha)$ for elliptical $(3 / \pi)$, rectangular, triangular, and trapezoidal sections. Solid symbols indicate further correlated values for rectangular and triangular sections employing $F_{i} \approx\left(1-\frac{1}{6} \sin 2 \alpha\right) / 3$.

$$
\left(A^{*}\right)_{t^{*}}=\left[A^{*} F_{i}\left(\frac{\bar{w}_{s}}{w_{s}}\right)\right]_{z^{*}}=\left[A^{*} F_{i}\left(\frac{\Delta_{s}}{\bar{\Delta}_{s}}\right) h_{z^{*}}^{*}\right]_{z^{*}},
$$

where it is observed that all interesting geometric dependence is contained within the ratio of global-to-local Laplacian scales. The expanded form of Eq. (24) is

$$
\begin{aligned}
{\left[\left(2 \lambda+h^{*} \tan \alpha\right) h^{*}\right]_{t^{*}}=} & \left\{\left(2 \lambda+h^{*} \tan \alpha\right) h^{*} F_{i}\left(\frac{\lambda+h^{*} \tan \alpha}{\lambda+\tan \alpha}\right)^{2}\right. \\
& \left.\times\left[\frac{1+(\lambda+\tan \alpha)^{2}}{h^{* 2}+\left(\lambda+h^{*} \tan \alpha\right)^{2}}\right] h^{* 2} h_{z^{*}}^{*}\right\}_{z^{*}},
\end{aligned}
$$

where again $\lambda \equiv a / H$. By inspection of Eq. (25), rectangular channel form (19) is recovered when $\alpha \rightarrow 0$ (or $h^{*} \tan \alpha / \lambda$ $\ll 1, \tan \alpha / \lambda \ll 1$ ), and triangular channel form (23) is recovered when $\lambda \rightarrow 0\left(h^{*} \tan \alpha / \lambda \gg 1, \tan \alpha / \lambda \gg 1\right)$.

$F_{i}\left(h^{*} ; \alpha, \lambda\right)$ for the trapezoidal section is computed numerically using Eqs. (3) and (4). Equation (3) is solved using MATLAB $\AA$ and the PDE toolbox ${ }^{8}$ and an adaptive mesh of $\sim 50000$ finite elements. The results are also plotted in Fig. 7 (dots) with the limiting values for the rectangular $(\alpha \rightarrow 0)$ and triangular $(\lambda \rightarrow 0)$ channel sections noted. Thus, in approximation, $F_{i}(\lambda=0)=1 / 6 \approx F_{i} \approx 1 / 3=F_{i}(\alpha=0)$ for all values of $\alpha, \lambda$, and $h^{*}$ for the trapezoidal channel flow.

Furthermore, it can be shown for such Cartesian scaled geometries that

$$
F_{i} \equiv \frac{F_{n}}{3} \frac{A_{\text {sect }}}{A_{\text {rect }}}=\frac{F_{n}}{3} \frac{A_{\text {trap }}}{A_{\text {rect }}}=\frac{F_{n}}{6} \frac{\left(2 \lambda+h^{*} \tan \alpha\right)}{\left(\lambda+h^{*} \tan \alpha\right)},
$$

where $A_{\text {sect }}$ is the local quadrant area of the section and $A_{\text {rect }}=\bar{x}_{s} \bar{y}_{s}$ is the scale quadrant area. The numerical values of $F_{i}$ in Fig. 7 are converted using $F_{n}$ from Eq. (26) and replotted in Fig. 9. "Worst case" limiting values for $F_{i}$ are represented in Fig. 10, revealing that the weak $h^{*}$ dependence is largely confined to $h^{*} \approx 0.1$. The collapse of the numerical data to $F_{n} \approx 0.97$ ( \pm 0.08 standard deviation for all values computed) favors the use of Eq. (26) and appears suitable for 


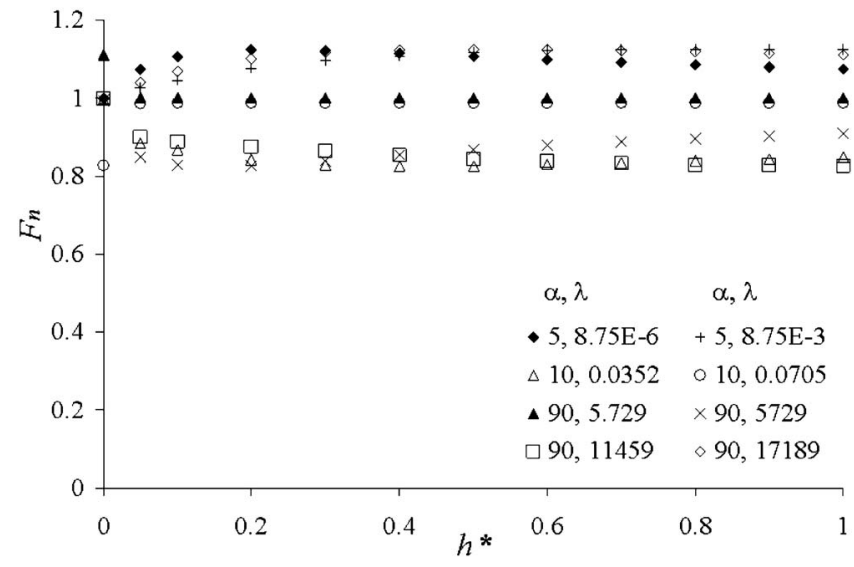

FIG. 10. Bounding values of $F_{n}\left(h^{*} ; \alpha, \lambda\right)$ for the trapezoidal section from Fig. 7 replotted against $h^{*}$. Values indicated for $\alpha=90^{\circ}$ are computed at $89.999^{\circ}$.

a family of duct sections possessing $x$ - and $y$-axis symmetry such as simple even-sided convex polygons and ellipses sketched in Fig. 11. It is also suitable for free surface flows where the free surface is coincident with zero shear stress symmetry planes. Substituting Eq. (26) into Eq. (25), the full evolution equation for the trapezoidal channel section is

$$
\begin{aligned}
{\left[\left(2 \lambda+h^{*} \tan \alpha\right) h^{*}\right]_{t^{*}} \approx } & \left\{\left(2 \lambda+h^{*} \tan \alpha\right) \frac{F_{n}}{6} \frac{\left(2 \lambda+h^{*} \tan \alpha\right)}{\left(\lambda+h^{*} \tan \alpha\right)}\right. \\
& \times\left(\frac{\lambda+h^{*} \tan \alpha}{\lambda+\tan \alpha}\right)^{2} \\
& \left.\times\left[\frac{1+(\lambda+\tan \alpha)^{2}}{h^{* 2}+\left(\lambda+h^{*} \tan \alpha\right)^{2}}\right] h^{* 3} h_{z^{*}}^{*}\right\}_{z^{*}}
\end{aligned}
$$

where $0.825 \leq F_{n}\left(h^{*} ; \alpha, \lambda\right) \leq 1.125$ and $F_{n} \approx 1$ might be a reasonable approximation. A concise assessment of the error incurred by taking $F_{n}=1$ is provided in Fig. 12 where exact numerical solutions to the steady solution of Eq. (27) are compared. The exact solutions employ a sixth order polynomial for $F_{i}$ and thus $F_{n}$ before solving Eq. (27) numerically. The agreement is excellent. For the worst case $a=0.02^{\circ}$ and $\lambda=3.49 \times 10^{-4}$, errors $<3 \%$ are typical with maximum errors $<7 \%$ near $z^{*}=1$ where the function $h^{*}$ becomes vertical. For the other more typical values of $\alpha$ shown, the scaled Laplacian and exact numerical solutions are essentially coincident.

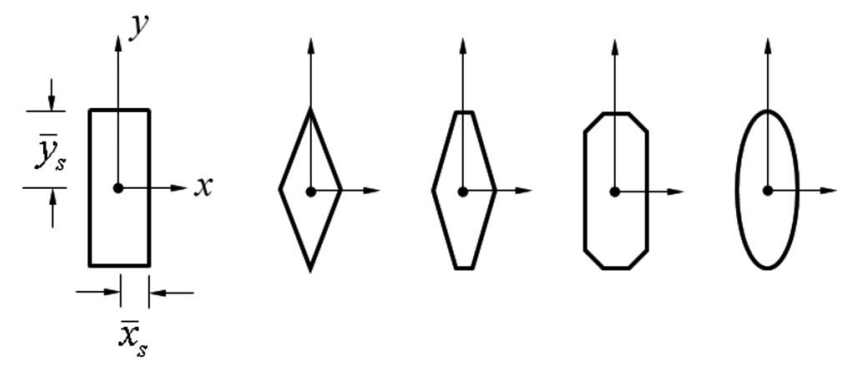

FIG. 11. Family of symmetric sections suitable for the Laplacian scale method.

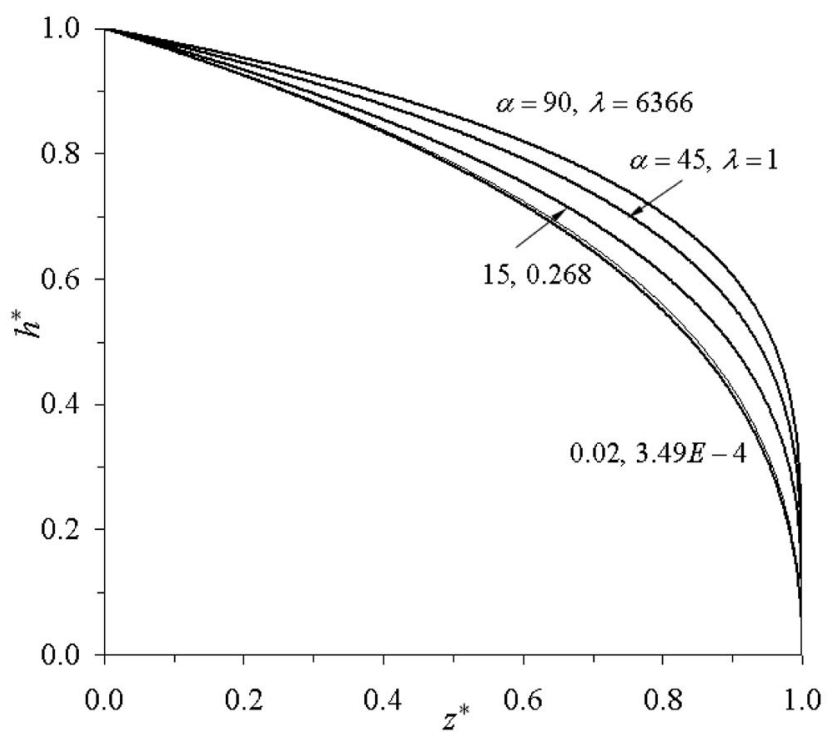

FIG. 12. Comparison of Laplacian scaled $\left(F_{n}=1\right.$, thin line) and exact numerical (heavy line) steady solutions to trapezoidal channel flow [Eq. (27)] for various values of $\alpha$ and $\lambda$. Curves are essentially coincident except for worst case condition $\alpha=0.02^{\circ}$ and $\lambda=3.49 \times 10^{-4}$, where errors are in general $<3 \%$ (note that $\alpha=89.999^{\circ}$ is implied by $\alpha=90^{\circ}$ on the plot).

Equation (27) is quickly obtained using the present method and embodies the majority of the sought geometric dependence of the flow. It serves as an adequate "starter" form for subsequent analysis. Such equation formulations are particularly helpful for analysis for more intricate geometries where limiting behavior may not be perceived a priori. The example of capillary-driven flow along interior corners that are rounded ${ }^{4}$ provides a case in point (see Fig. 13). Such free surface flows can possess a variety of limiting behaviors for a single geometry and require fully numerical solutions. Nonetheless the Laplacian method quickly captures the correct form and order of terms in a governing evolution equation facilitating theoretical understanding as well as numerical setup, solution, and benchmark.

\section{IMPLICATIONS OF LAPLACIAN SCALE TO POISEUILLE NUMBER}

As briefly mentioned in Sec. I A, the Laplacian scaling method characterizes the geometric dependence of viscous diffusion with greater accuracy than the hydraulic diameter scaling. It does so by characterizing the effective viscous

(a)

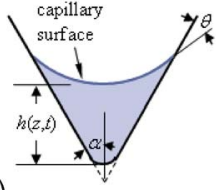

FIG. 13. (Color online) Example of capillary-driven flow along a rounded interior corner: (a) The fully numeric flow is readily reduced to analytic forms by the Laplacian scaling method (Ref. 4), (b) sharp corner, (c) rectangular domain, and (d) thin film flow. 
length with greater accuracy. If one exploits these benefits to compute Fanning friction factors $f$ for such flows (Fig. 11) one might define a Laplacian diameter,

$$
D_{\Delta} \equiv\left(\frac{F_{i}}{\bar{\Delta}_{s}}\right)^{1 / 2}=\left(\frac{F_{n} A_{s}}{3\left(\bar{T}_{s x y}+\bar{T}_{s x y}^{-1}\right)}\right)^{1 / 2}
$$

and a Laplacian Reynolds number, $\operatorname{Re}_{\Delta}=\rho U D_{\Delta} / \mu$, to find that

$$
2.67 \ldots \leq \mathrm{Po}_{\Delta} \equiv f \mathrm{Re}_{\Delta} \leq 2 \sqrt{3} .
$$

This result implies that the Laplacian Poiseuille number, $\mathrm{Po}_{D} \approx 3( \pm 10 \%)$, is a weak $O(1)$ function of the section geometry and may be treated approximately as a constant for laminar flows. As a consequence, $f \approx 3 / \mathrm{Re}_{D}$ for all applicable cross sections. This compares to $14.2 \ldots \leq \mathrm{Po} \equiv f \mathrm{Re}$ $\leq 24$ for traditional laminar flows, where $\mathrm{Re}$ is scaled on $D_{\text {hyd }}$.

\section{CONCLUDING REMARKS}

In summary, the Laplacian scaling method amounts to nothing more than treating the operator as its own geometric scale quantity, $\bar{\Delta}_{s}$. It appears to be a quick and effective method for certain predominately 2D fields with possibilities for weak three-dimensional as well as temporal dependence. The subsequent process of nondimensionalization yields a modified 2D Poisson equation with $z$ - and $t$-dependent variables and coefficients [Eq. (3)], which when solved for the dimensionless area-averaged velocity $\left\langle\bar{w}^{*}\right\rangle \equiv F_{i}[$ Eq. (4)] possesses a weak dependence on numerical data-a dependence that is further reduced when $F_{n}$ from Eq. (26) is employed. In such situations exact or approximate average quantities are readily determined as are the subsequent equations that depend on them.

\section{ACKNOWLEDGMENTS}

This work is supported in part by the National Aeronautics and Space Administration through Grant No. NNC05AA29A and National Science Foundation Grant No. CTS-0521890. M.M.W. would like to thank A. Bejan for an introduction to scale analysis and G. Recktenwald for a push.

${ }^{1}$ W. B. Krantz, Scaling Analysis in Modeling Transport and Reaction Processes (Wiley, Hoboken, NJ, 2007).

${ }^{2}$ R. K. Shah and A. L. London, Laminar Flow Forced Convection in Ducts (Academic, London, 1978).

${ }^{3}$ A. Bejan, Convection Heat Transfer, 3rd ed. (Wiley, New York, 2004).

${ }^{4}$ Y. Chen, M. M. Weislogel, and C. Nardin, "Capillary-driven flows along rounded interior corners," J. Fluid Mech. 566, 235 (2006).

${ }^{5}$ P. S. Ayyaswamy, I. Catton, and D. K. Edwards, "Capillary flow in triangular groves," J. Appl. Mech. 41, 332 (1974).

${ }^{6}$ T. C. Ransohoff and C. J. Radke, "Laminar flow of a wetting liquid along corners of a predominately gas-occupied noncircular pore," J. Colloid Interface Sci. 121, 392 (1988).

${ }^{7}$ F. M. White, Viscous Fluid Flow, 3rd ed. (McGraw-Hill, New York, 2006), p. 117, Fig. 3-13.

${ }^{8}$ D. Bolleddula, "Capillary flow analysis and computation of dewetting and wetting resistances in angular geometries," M.S.M.E. thesis, Portland State University, 2007. 
Physics of Fluids is copyrighted by the American Institute of Physics (AIP). Redistribution of journal material is subject to the AIP online journal license and/or AIP copyright. For more information, see http://ojps.aip.org/phf/phfor.jsp 